\title{
Déclaration concernant le poliovirus et les voyageurs internationaux du Comité consultatif de la médecine tropicale et de la médecine des voyages (CCMTMV)
}

\author{
Comité consultatif de la médecine tropicale et de la médecine des voyages
}

*Auteur-ressource : chui@cheo.on.ca

\section{Résumé structuré}

Introduction : Le 5 mai 2014, l'Organisation mondiale de la Santé a déclaré la récente propagation internationale du poliovirus sauvage urgence de santé publique de portée internationale. Des recommandations temporaires ont été publiées afin de réduire le risque de nouvelle propagation de la maladie. Celles-ci visent particulièrement les pays qui exportent actuellement le poliovirus sauvage et ceux qui sont infectés par le poliovirus sauvage mais qui ne l'exportent pas. La présente déclaration a pour objet d'examiner les répercussions de ces nouvelles recommandations temporaires pour les voyageurs canadiens et de fournir des lignes directrices à l'intention des professionnels de la santé pour la prévention de la poliomyélite (polio) chez les voyageurs canadiens.

Méthodes : Cette déclaration a été préparée par le Comité consultatif de la médecine tropicale et de la médecine des voyages (CCMTMV) et se veut un complément au Guide canadien d'immunisation, une ressource exhaustive sur l'immunisation. Le CCMTMV a pris en considération la nécessité de protection ainsi que les effets indésirables potentiels de la vaccination.

Contexte : La poliomyélite est une maladie virale très contagieuse évitable par la vaccination. Asymptomatique dans la majorité des cas, la poliomyélite s'attaque au système nerveux central et entraîne une paralysie dans environ $1 \%$ des cas.

Épidémiologie et risque pour le voyageur : En 1994, le Canada a été déclaré exempt de poliovirus sauvage. Les cas survenus depuis lors ont été associés à des cas importés et, dans de rares cas ( 1 pour 2,7 millions de doses), à la poliomyélite paralytique contractée à la suite de l'administration du vaccin antipoliomyélitique oral (VPO). II existe toujours un risque de contracter la maladie pour les voyageurs qui se rendent dans des régions où la poliomyélite n'est pas éradiquée.

Prévention : La vaccination systématique des enfants et des adultes est recommandée au Canada. La version la plus récente du Guide canadien d'immunisation présente des renseignements complets sur les recommandations concernant le vaccin contre la poliomyélite au Canada. Les voyageurs doivent s'assurer qu'ils sont vaccinés contre la poliomyélite avant de se rendre dans des pays où l'on sait ou soupçonne qu'il y a circulation du poliovirus.

\section{Introduction}

Le 5 mai 2014, l'Organisation mondiale de la Santé (OMS) a déclaré la récente propagation internationale du poliovirus sauvage urgence de santé publique de portée internationale $(1,2)$. Cette déclaration faisait suite aux éclosions de poliomyélite dues aux cas importés dans plusieurs pays en 2013 où la maladie avait été éradiquée. En 1988, une initiative mondiale visant l'éradication de la poliomyélite pour l'an 2000 a été mise en œuvre à la suite de l'adoption d'une résolution par l'Assemblée mondiale de la Santé. La poliomyélite était alors endémique dans 125 pays sur cinq continents. C'est ainsi que l'Initiative mondiale pour l'éradication de la poliomyélite (disponible en anglais seulement) est née, sous la direction des gouvernements nationaux, del'OMS, du Rotary 
International, des Centers for Disease Control and Prevention et du Fonds des Nations Unies pour l'enfance. En 1999, la poliomyélite de type 2 avait été éradiquée à l'échelle mondiale. Au début de 2013, le nombre des cas de poliomyélite avait diminué de plus de $99 \%$, les trois pays d'endémie restant étant l'Afghanistan, le Nigéria et le Pakistan $(3,4)$.

En 2013, la République arabe syrienne, le Cameroun et un certain nombre de pays de la Corne de l'Afrique, dont la Somalie, l'Éthiopie et le Kenya, présentaient des éclosions de poliomyélite, par suite de l'importation de pays où la maladie était endémique (4). En outre, le virus a été détecté dans les échantillons d'eaux usées en Israël, en Cisjordanie et dans la bande de Gaza, mais aucun cas n'a été signalé à ce jour.(4). Des cas de poliomyélite attribuables à la circulation du poliovirus dérivé d'une souche vaccinale ont été signalés en 2013 au Nigéria, au Niger, au Tchad, au Cameroun, au Pakistan, en Afghanistan, en Somalie et au Yémen.

En vue de limiter une nouvelle propagation de la poliomyélite, une intervention internationale coordonnée a été jugée essentielle. Les recommandations temporaires concernant la vaccination antipoliomyélitique ont été publiées en application du Règlement sanitaire international 2005 et seront évaluées dans un délai de trois mois. Celles-ci visent particulièrement les pays qui exportent actuellement le poliovirus sauvage et ceux qui sont infectés par le poliovirus sauvage mais qui ne l'exportent pas (1,2). Ces recommandations comprennent des directives sur la vaccination avant d'entreprendre des voyages internationaux pour les personnes résidant ou effectuant un séjour de longue durée dans ces pays (pour la description complète, se reporter à la référence (1)). Le site Web de l'Initiative mondiale pour l'éradication de la poliomyélite (disponible en anglais seulement) présente notamment la liste des pays où des cas confirmés d'infection à poliovirus sauvage sont signalés ainsi que des mises à jour hebdomadaires sur la situation épidémiologique et la répartition du nombre de cas de poliovirus sauvage par pays. L'Agence de la santé publique du Canada présente également des conseils de santé aux voyageurs à jour, de même que la situation mondiale de la polio (5). La présente déclaration a pour objet d'examiner les répercussions de ces nouvelles recommandations temporaires pour les voyageurs canadiens et de fournir des lignes directrices à l'intention des professionnels de la santé pour la prévention de la poliomyélite chez les voyageurs canadiens.

\section{Méthodes}

Cette déclaration a été élaborée par un groupe de travail du Comité consultatif de la médecine tropicale et de la médecine des voyages (CCMTMV). Chaque membre est bénévole, et aucun d'entre eux n'a signalé de conflit d'intérêts pertinent. La déclaration a été élaborée comme complément à la révision approfondie de la documentation et à l'analyse effectuée pour formuler les recommandations du Comité consultatif national de l'immunisation, présentées dans le Guide canadien d'immunisation, une ressource exhaustive sur l'immunisation. Le CCMTMV a pris en considération la nécessité de protection, les exigences en matière de vaccination et les effets indésirables potentiels de la vaccination.

\section{Contexte}

La poliomyélite est une maladie virale extrêmement contagieuse, évitable par la vaccination, qui est causée par l'un des trois sérotypes de poliovirus (types 1, 2 et 3). Aujourd'hui, la poliomyélite est causée principalement par le type 1, car le type 2 a été éradiqué à l'échelle mondiale en 1999 et le type 3 a été ramené à son plus bas niveau. La manifestation clinique est plus fréquente chez les enfants de moins de cinq ans; cependant, toute personne non immunisée contre le poliovirus, peu importe son âge, peut contracter l'infection. La poliomyélite se transmet d'une personne à une autre, principalement par voie fécale-orale. La transmission par contact étroit avec les sécrétions ou la salive d'une personne infectée est également possible, mais rare.

La plupart des personnes infectées ne présentent aucun symptôme, mais dans environ 1 \% des cas, la poliomyélite peut s'attaquer au système nerveux central et détruire les cellules nerveuses qui font fonctionner les muscles, ce qui entraîne la paralysie. La plupart des cas de poliomyélite sont causés par le virus qui circule naturellement dans l'environnement (virus de type sauvage). Dans de très rares cas, l'infection peut être causée 
par les souches du virus présentes dans le vaccin antipoliomyélitique oral (VPO). Lorsqu'elle est contractée, l'infection par la souche vaccinale du virus (poliomyélite paralytique associée à la vaccination) se produit généralement après l'administration du VPO (environ 1 pour 2,7 millions de doses), mais peut parfois survenir chez les contacts étroits. Le (VPO) n'est pas offert sur le marché canadien, bien qu'il continue d'être largement utilisé à l'échelle internationale. Le vaccin antipoliomyélitique inactivé (VPI), qui est utilisé au Canada, ne cause pas d'infection ni la poliomyélitique paralytique associée à la vaccination.

Le VPIse compose de souches inactivées des trois types de poliovirus. Ce vaccin déclenche une excellente réponse immunitaire protectrice chez la plupart des patients. Cependant, comme le vaccin n'induit qu'une très faible immunité mucosale, le poliovirus administré peut tout de même se multiplier et être excrété dans les matières fécales des personnes immunisées.

Une description complète des vaccins antipoliomyélitiques homologués au Canada peut être obtenue dans le Guide canadien d'immunisation (6).

\section{Épidémiologie de la poliomyélite au Canada}

En 1994, l'OMS a déclaré le Canada exempt de poliovirus sauvage $(7,8)$. Au Canada, le dernier cas de poliovirus sauvage est survenu en 1977 (6). Les cas de poliomyélite signalés depuis lors au Canada ont été associés à des cas importés d'infection à poliovirus sauvage et à l'utilisation du VPO. Entre 1980 et 1995, 11 cas de poliomyélite paralytique associée à l'administration du VPO ont été signalés au Canada (6). En 1995 et 1996, tous les programmes de vaccination ont abandonné le VPO pour utiliser le VPI. Une description complète de l'épidémiologie de la poliomyélite au Canada est présentée dans le Guide canadien d'immunisation (6).

\section{Risque pour les voyageurs}

Jusqu'à ce qu'on ait déclaré officiellement l'éradication de la poliomyélite à l'échelle mondiale, il y aura un risque pour les voyageurs. II existe peu ou pas de risques pour les personnes qui se rendent dans des régions où la poliomyélite a été éliminée, comme en Amérique, en Europe et dans les pays du Pacifique occidental. Le risque pour les voyageurs qui se rendent dans d'autres régions est faible, mais dépend de facteurs tels que la durée du séjour, les conditions de vie ainsi que la salubrité de l'eau et des aliments du pays visité.

\section{Prévention}

\section{Recommandations en matière de vaccins pour les voyageurs canadiens}

Peu importe le programme de voyage, la vaccination systématique des enfants et des adultes est recommandée au Canada. Chez les enfants, on recommande une série primaire de vaccins à 2, 4 et 6 mois ainsi qu'entre 12 et 23 mois (habituellement à 18 mois) et une dose de rappel entre 4 et 6 ans. Des renseignements complets sur les recommandations concernant le vaccin antipoliomyélitique au Canada sont présentés dans la version la plus récente du Guide canadien d'immunisation, et les calendriers d'immunisation provinciaux et territoriaux sont affichés sur le site Web de l'Agence de la santé publique du Canada (6).

Le vaccin antipoliomyélitique inactivé (VPI) est le seul vaccin offert actuellement au Canada.

Pour les voyageurs qui se rendent dans des pays où l'on sait qu'il y a circulation du poliovirus ou dans des pays qui présentent un risque d'importation de la poliomyélite :

Il est conseillé aux personnes qui voyagent avec des enfants ou des nourrissons non immunisés de sérieusement envisager de reporter leur voyage dans les régions où la poliomyélite est endémique ou dans les régions où des éclosions surviennent, et ce, idéalement jusqu'à l'immunisation complète des enfants ou des nourrissons non immunisés ou jusqu'à ce qu'ils aient reçu au moins deux doses du VPI (taux de séroprotection de $90 \%$, se reporter à la section Immunogénicité et innocuité du vaccin). 


\section{Nourrissons}

1) Lorsqu'une protection rapide est nécessaire, la première dose du vaccin DCaT-VPI-Hib ou du vaccin DCaT-HB-VPI-Hib peut être administrée à l'âge de 6 semaines.

2) On peut donner les trois premières doses à intervalles de 4 semaines.

3) Idéalement, la quatrième dose devrait être donnée 12 mois après la troisième dose.

4) Dans certaines situations (p. ex. voyage), la quatrième dose peut être administrée au minimum 6 mois après la troisième dose; il faut toutefois que l'enfant soit âgé de 12 mois ou plus pour assurer une immunité prolongée.

5) Si le VPI n'est pas disponible dans la région où le nourrisson doit se rendre, on peut compléter la série vaccinale en utilisant le VPO. Les parents des nourrissons qui reçoivent le VPO devraient être informés que les bébés peuvent excréter le poliovirus pendant quelques semaines après la vaccination (se reporter à la section Immunogénicité et innocuité du vaccin); la vaccination antipoliomyélitique des contacts familiaux et des personnes qui s'occupent de ces bébés devrait donc être à jour. Les personnes qui s'occupent de ces bébés devraient également être informées de l'importance de bien se laver les mains après le changement de couches. Le risque de poliomyélite paralytique associée à la vaccination est faible (environ 1 pour 2,7 millions de doses distribuées).

6) Si le voyage ne peut être reporté, les risques d'un programme de vaccination incomplet avant le départ doivent être communiqués aux parents (se reporter à la section Immunogénicité et innocuité du vaccin).

\section{Enfants non immunisés}

1) Les enfants non immunisés devraient entreprendre une série primaire d'un vaccin contenant le VPI, et la possibilité d'adopter un calendrier accéléré (consulter le Calendrier) (6).

\section{Voyageurs non immunisés ou non complètement immunisés}

Les voyageurs qui ne sont pas immunisés ou qui ne le sont pas complètement devraient recevoir un vaccin contenant le VPI qui convient à leur âge (se reporter au Guide canadien d'immunisation).

Les enfants qui ont reçu une série primaire complète n'ont pas à recevoir d'autres doses du VPI avant un voyage.

Dans le cas des adultes qui ont reçu une série primaire complète, l'administration d'une dose unique d'un vaccin à composant antipoliomyélitique est recommandée.

\section{Recommandations temporaires concernant les voyages à l'étranger}

1. Les recommandations sont fondées sur la déclaration de l'urgence de santé publique de portée internationale de l'Organisation mondiale de la Santé. Ces recommandations visent à prévenir la propagation de la poliomyélite; leur objectif principal n'est pas la protection du voyageur.

Les voyageurs qui ont l'intention d'effectuer un séjour de longue durée (plus de 4 semaines) dans l'un des pays d'où le poliovirus est exporté ou dans l'un des pays où le poliovirus est présent doivent se conformer aux récentes recommandations de l'OMS, c'est-à-dire recevoir une dose de vaccin antipoliomyélitique oral (VPO) ou de vaccin antipoliomyélitique inactivé (VPI) dans un délai de 4 semaines à 12 mois avant un voyage international. Par conséquent, pour qu'une personne puisse sortir de l'un de ces pays ou entrer dans un autre, elle devra vraisemblablement recevoir une dose de 
rappel du vaccin antipoliomyélitique, même si elle a déjà reçu la dose de rappel à l'âge adulte et qu'elle est considérée comme adéquatement immunisée.

Les pays qui exportent actuellement le poliovirus sauvage sont le Pakistan, la Guinée équatoriale, le

Cameroun et la République arabe syrienne et les pays où le poliovirus sauvage est présent actuellement mais qui ne l'exportent pas sont l'Afghanistan, I'Éthiopie, I'Iraq, Israël, la Somalie et, plus particulièrement, le Nigéria. L'OMS a élaboré une foire aux questions pour les voyageurs (disponible en anglais seulement) au sujet des recommandations temporaires (9).

\section{Voyage en Inde}

Le ministère de la Santé et du Bien-être social de l'Inde (disponible en anglais seulement) a dressé sa propre liste de pays où la poliomyélite est endémique et de pays où des cas importés de poliomyélite ont été signalés et a publié des exigences pour les visiteurs originaires de ces pays précisant que ces derniers doivent présenter une preuve de vaccination par le VPO avant d'entrer en Inde. Les résidents indiens doivent présenter une preuve de vaccination par le VPO avant de voyager dans ces pays (10).

Ces exigences ne sont pas obligatoires pour les citoyens canadiens (c.-à-d. ressortissants étrangers) ni les voyageurs canadiens, tant pour entrer en Inde et en sortir que pour un transit dans des pays où le poliovirus est présent.

\section{Carnet de vaccination contre la poliomyélite}

On recommande aux voyageurs visés par ces recommandations temporaires d'apporter un carnet de vaccination écrit dans le cas où une preuve de vaccination serait demandée pour satisfaire aux exigences d'entrée et de sortie (11). Selon le Règlement sanitaire international 2005, le Certificat international de vaccination ou de prophylaxie est le document officiel pour montrer une preuve de vaccination contre la poliomyélite. On peut se le procurer actuellement dans les Centres de vaccination contre la fièvre jaune au Canada. Le site Web de l'Agence de la santé publique du Canada présente la liste complète de ces centres.

L'Agence de la santé publique du Canada a élaboré des lignes directrices pour remplir le $\underline{\text { Certificat }}$ international de vaccination ou de prophylaxie.

\section{Immunogénicité et innocuité du vaccin}

Des études ont montré que plus de 89 à $100 \%$ des nourrissons ayant reçu le VPI selon un calendrier à deux doses (à l'âge de 2 et 4 mois) et de 93 à $100 \%$ des nourrissons ayant reçu le vaccin selon un calendrier à trois doses (à l'âge de 2, 3 et 4 mois) ont développé des anticorps séroprotecteurs un mois après l'administration de la dernière dose. À titre de comparaison, le taux de séroprotection est de près de $100 \%$ selon un calendrier d'administration du vaccin à 2, 4, 6 mois et entre 12 et 18 mois (12).

Les effets indésirables qui se manifestent après l'administration du VPI se limitent généralement à des réactions bénignes au point d'injection. Les effets indésirables graves sont rares après l'administration des vaccins contenant le VPI et, dans la plupart des cas, les données sont insuffisantes pour établir un lien de causalité. On a déjà observé des réactions anaphylactiques, mais seulement dans de très rares cas (6).

Après l'immunisation par le VPO, les souches vaccinales du poliovirus peuvent persister dans la gorge pendant une à deux semaines, et dans les matières fécales pendant plusieurs semaines. Dans de rares cas, et notamment chez les personnes immunodéprimées, le poliovirus (résultant d'une infection naturelle ou de l'administration du VPO) peut être excrété pendant de longues périodes (allant de plus de six mois à quelques années). Une description complète de l'innocuité du vaccin est présentée dans le Guide canadien d'immunisation. 
Les personnes non immunisées peuvent être «passivement immunisées » lorsqu'elles entrent en contact avec les souches du VPO, généralement dans les matières fécales. L'immunisation par le VPI n'entraîne pas l'excrétion des souches vaccinales.

\section{Voyageurs immunodéprimés}

Le VPI peut être administré aux voyageurs immunodéprimés. Le VPO ne doit pas être administré aux personnes immunodéprimées qui prévoient effectuer un séjour de plus de quatre semaines dans un pays qui exige une dose de rappel pour quitter. Si le VPO est le seul vaccin offert dans ce pays, il pourrait s'avérer utile que ces personnes aient une lettre d'exemption.

\section{Interventions en matière d'hygiène}

On recommande aux voyageurs d'adopter de bonnes mesures d'hygiène, dont le lavage fréquent des mains, afin de réduire le risque de transmission de la poliomyélite lors d'un voyage.

\section{Références}

(1) Organisation mondiale de la Santé. 2014. Déclaration de l'OMS suite à la réunion du Comité d'urgence du Règlement sanitaire international concernant la propagation internationale du poliovirus sauvage. http://www.who.int/mediacentre/news/statements/2014/polio20140505/fr/. [consulté le 14 mai 2014].

(2) Global Polio Eradication Initiative. Polio News, Special Alert. 2014. http://www.polioeradication.org/Portals/O/Document/Media/Newsletter/PN201404 EN.pdf. [consulté le 20 mai 2014].

(3) Initiative mondiale pour l'éradication de la poliomyélite. Plan Stratégique pour l'éradication de la poliomyélite et la phase finale 2013-2018. Genève (Suisse) : OMS, 2013.

(4) Organisation mondiale de la Santé. Note de synthèse de I'OMS sur les vaccins antipoliomyélitiques - Janvier 2014. Relevé épidémiologique hebdomadaire (28 février 2014); 89(9):73-92.

(5) Agence de la santé publique du Canada. Polio (poliomyélite) : situation mondiale. http://voyage.gc.ca/voyager/sante-securite/conseils-sante-voyageurs/polio. [consulté le 20 mai 2014].

(6) Agence de la santé publique du Canada. Guide canadien d'immunisation. Cat. : HP40-3/2014F éd., Ottawa (Ontario) : Agence de la santé publique du Canada; 2014.

(7) Robbins FC, de Quadros CA. Certification of the eradication of indigenous transmission of wild poliovirus in the Americas. J Infect Dis. février 1997 ; 175 (Suppl 1):S281-5.

(8) Working Group on Polio Eradication, Bentsi-Enchill A. Protocol for the investigation of acute flaccid paralysis and suspected paralytic poliomyelitis. Paediatr Child Health 1997; 2(6):409-412.

(9) World Health Organization. 2014. Frequently Asked Questions for Travellers. http://www.polioeradication.org/Portals/0/Document/Emergency/FAQs travellers.pdf. [consulté le 20 mai 2014].

(10) Ministry of Health and Family Welfare, Government of India. Requirements of polio vaccination for international travellers between India and polio infected countries. 2014. http://mohfw.nic.in/WriteReadData/I892s/8285260748Requirement\%20of\%20Polio\%20vaccination\%20for \%20International\%20travellers\%20between\%20India\%20\&\%20polio\%20infected\%20countries.pdf. [consulté le 15 mai 2014]. 
(11) Organisation mondiale de la Santé. Voyages internationaux et santé. Genève (Suisse) : Organisation mondiale de la Santé; 2012.

(12) Plotkin S, Vidor E. Poliovirus Vaccine (Inactivated). In: Plotkin S, Orenstein W, Offit P, éditeurs. Vaccines. $5^{\mathrm{e}}$ éd. Philadelphia: Saunders; 2008.

\section{Remerciements}

La présente déclaration a été préparée par le groupe de travail sur la poliomyélite : Hui C., Teitelbaum P., Bui Y. G. et Geduld, J.

Le CCMTMV tient à remercier Kelsie Jagt et Tanya Straight-Caron pour leur contribution à la déclaration.

Membres du CCMTMV : Boggild A., Brophy J., Bui Y. G., Crockett M., Ghesquiere W., Greenaway C., Henteleff A., Libman M., Teitelbaum P. et McCarthy A. (présidente)

Représentants chargés de la liaison : Hui C. (Société canadienne de pédiatrie) et Gershman M. (Centers for Disease Control and Prevention [États-Unis.]).

Membres d'office : Marion D. (Centre des services de santé des Forces canadiennes, ministère de la Défense nationale), McDonald P. (Division des médicaments anti-infectieux, Santé Canada), Schofield S. (Direction de la protection de la santé de la Force, ministère de la Défense nationale) et Tepper M. (Direction de la protection de la santé de la Force, ministère de la Défense nationale)

Membre émérite : Jeanes C. W. L.

\section{Conflit d'intérêts}

Aucun déclaré 


\section{Actualités sur les maladies infectieuses}

Brefs résumés d'articles récemment publiés au sujet de maladies infectieuses

\section{Management of chronic Hepatitis B infection: Current treatment guidelines, challenges, and new developments [Prise en charge de l'infection chronique par le virus de hépatite $B$ : Les lignes directrices actuelles en matière de traitement, les défis et les nouvelles réalités] \\ Les lignes directrices internationales actuelles recommandent comme traitement de première intention de l'infection chronique par le virus de l'hépatite B (VHB) l'administration de PEG-Interféron (entécavir ou ténofovir), mais le traitement optimal est controversé. Des données probantes de plus en plus nombreuses semblent pencher en faveur du recours au génotypage du VHB ainsi qu'à la réalisation, en cours de traitement, de mesures sérielles de l'antigène de surface HB et de la cinétique de l'ADN viral dans le but de prédire et de surveiller l'évolution de la réponse au traitement antiviral. La prolongation du traitement et le recours à une polythérapie pourraient permettre d'augmenter la probabilité d'obtenir une réponse virologique soutenue.}

Tang C, On Yau T, Yu J, World J Gastroenterol 28 mai 2014; 20(20): 6262-6278

Les membres du Comité consultatif de la médecine tropicale et de la médecine des voyages (CCMTMV) pleurent la mort de C. William (Bill) Jeanes.

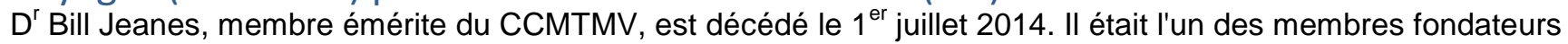
du CCMTMV et a participé à ses réunions pendant 24 années. L'étendue et l'ampleur de son expérience et de sa vision nous manqueront.

http://www.legacy.com/obituaries/ottawacitizen/obituary.aspx?n=c-william-I-jeanes-bill\&pid=171615810 